East African Medical Journal Vol. 81 No. 6 June 2004

STRATEGIC MANAGEMENT OF TECHNOLOGY IN PUBLIC HEALTH SECTOR IN KENYA AND SOUTH AFRICA

M. Ogembo-Kachieng'a, PhD Biomed(Eng), BCom(Hons) Finance, MSc (Distinction) Elec. Eng. C.Eng (UK), Pr. Eng(SA), Professor of Technological Entrepreneurship, Department of Engineering and Technology Management, Universiy of Pretoria, South Africa and W. O. Ogara, PhD, Department of Public Health, Pharmacology and Toxicology, Faculty of Veterinary Sciences, University of Nairobi, P.O. Box 29053, Nairobi, Kenya

Request for reprints to: Prof. M. Ogembo-Kachieng'a, Department of Engineering and Technology Management, University of Pretoria, Main Campus, Pretoria 0001, South Africa

\title{
STRATEGIC MANAGEMENT OF TECHNOLOGY IN PUBLIC HEALTH SECTOR IN KENYA AND SOUTH AFRICA
}

\author{
M. OGEMBO-KACHIENG'A and W. O. OGARA
}

\begin{abstract}
Objective: To investigate factors contributing to health care equipment problems and associated technological investments in public hospitals.

Design: The article reviews the processes of equipment planning, procurement and management. Analysis of results and observations from experts leads to recommendations and suggestions on good equipment management practices in public hospitals. It also provides recommendations for competitive utilisation of equipment in the public health sector. Settings: Health care equipment management in Kenya and South Africa in 1999/2000. Institutions: A total of 10 public equipment maintenance institutions, and 38 equipment maintenance experts participated in the survey. Majority of the participants were drawn from teaching hospitals.

Results: It is evident that the way health technology is managed in health care institutions directly affects the quality of treatment patients receive. Although strategic importance of technology in health care has been documented widely in scientific literature; equipment planning, procurement and management have not received the attention they deserve in the transformation of health care services in the two countries under the survey.

Conclusions: The growing demand for more and better health care greatly expands the role of health care equipment in the delivery of health services. Kenya and South Africa have tried various strategies to improve access, quality and cost-efficiency in the health care delivery systems. However it is clear that the optimal method has yet to be found. Good management of health care equipment increases efficiency in health care services and enhances health outcomes.
\end{abstract}

\section{INTRODUCTION}

Technological innovations in the 21 st century have reshaped the field of medicine and the delivery of health care services. Although the art of medicine has a long history, recent advances in health technology have provided a wide range of diagnostic, therapeutic, and rehabilitative tools and instruments that are now routinely used in the cure of specific disease and illness. In the process, modern hospitals have evolved as technologically sophisticated health care facilities serviced by technologically specialised personnel.

Innovations and developments in health technology have contributed significantly to the quality of health care provided by various health facilities, but have also brought new challenges in management of health care services for both developing and developed countries. These include the development of the necessary infrastructure and human resources required to plan, deploy, manage and assess new technologies, and also the development of strategies required to manage cost containment.
Health service planners, hospital administrators, physicians and other health care professionals need to understand the forces that add value to cost-effectiveness and efficiency of health care delivery systems. Because technology and health care equipment plays such a significant role in health care services, it is vital to understand the role of technology management in order to be able to communicate effectively about it to health planners. There is not only a need for appropriate equipment. Suitable infrastructural arrangements are also required in order to make the role of technology more competitive and clinically efficacious. Effective management of technology directly contributes to improved patient health outcomes. Selecting and procuring health care equipment, which is appropriate, efficient and safe, remains a major challenge to the Kenyan and South African governments.

Literature review: Health technology management has been defined as an accountable, systematic approach to ensuring that cost-effective, efficacious, safe, and appropriate equipment is available to meet the demands 
of quality care(1). In most hospitals in industrialised countries, the importance of technology management, especially equipment maintenance, is well recognised as an integral part of hospital management.

Equipment asset management encompasses the traditional duties of a clinical engineering department. This includes involvement in equipment acquisition and other life cycle issues, supplying equipment information and training, monitoring and evaluating equipment, and documenting equipment(2-4).

It also includes using equipment management information systems to provide a more complete analysis of specific financial and clinical indicators, as well as for compliance with the regulatory and accreditation requirements(2). Viewed from this perspective, technology management is actually the practice of integrating technology strategy with the health service strategy of the hospital, in the most efficient and costeffective way(4-6). It is an accepted norm in developed countries that technology forms an integral part of health policy and planning. In many hospitals in these countries, health care equipment maintenance and management have become the responsibility of the clinical/medical engineering department $(2,4)$. In contrast, in many developing, emerging economies, like Kenya and South Africa, health care equipment still remains a major problem in the delivery of health services $(4,7)$.

In addition, equipment tender boards in Kenya and South Africa lack technical expertise to select equipment that meets health needs and technology requirements. A study on equipment procurement in Kenya and South Africa found that the tender boards had no technology experts as members $(6,8,9)$. Lacking information about efficacy, safety, cost-effectiveness and cost of ownership of technology, the tender boards used equipment purchase price as a major guide, rather than returns on investment (ROI) as should be the case.

A review of the literature on equipment maintenance problems in sub-Saharan Africa shows that earlier studies $(4,10)$ were project-oriented and focused on individual, donor-supported programmes. This is the first study exclusively focused on equipment maintenance personnel. It seeks to investigate equipment maintenance problems from the point of view of clinical engineers and technology managers in public hospitals.

\section{MATERIALS AND METHODS}

Survey of health care equipment maintenance specialists: Between September 1999 and September 2000, a number of health care equipment maintenance specialists were interviewed to determine current maintenance practices in Kenya and South Africa. The equipment maintenance experts were drawn from small (75-300 beds), medium (301-600 beds) and large (>600 beds) hospitals, as well as regional equipment maintenance workshops. The categorisation of hospitals by bed size provided a more realistic and universal measure than administrative classification, such as district or provincial hospitals.
Survey rationale: The purpose of this survey of equipment maintenance experts was to"ënvestigate the factors contributing to health care equipment maintenance problems in public health institutions. First hand professional views and information from practising technicians, clinical engineers and technology in small, medium and large public hospitals, as well as regional equipment maintenance workshops were used.

Survey organisation: Fifty six questionnaires were mailed to targeted technology managers, clinical/medical engineers and technicians (equipment maintenance specialists) in public hospitals in Kenya and South Africa; 38 responses were received (68\% response). The respondents included four equipment specialists from small hospitals, 10 from medium hospitals, 16 from large hospitals, and eight from regional equipment maintenance workshops. The responses from three other facilities were incomplete and therefore not included in the survey.

The majority of respondents were drawn from urban teaching hospitals, because it has been reported that teaching hospitals invest roughly six times as much in technology annually as non-teaching hospitals, and urban hospitals invest about four times as much as their rural counterparts(6). The number of participants (experts) are summarised according to job title in Table 1. The average working experience of participants was 5.53 years.

\section{Table 1}

Number of participants by job title

\begin{tabular}{ll}
\hline Job title & $\begin{array}{l}\text { No. of participants } \\
\text { (experts) }\end{array}$ \\
\hline Equipment maintenance manager & 4 \\
Clinical engineers & 4 \\
$\begin{array}{ll}\text { Clinical Engineering Technicians } \\
\text { (Med./Biomed. Eng. Technicians) }\end{array}$ & 30 \\
\hline Total & 38 \\
\hline
\end{tabular}

Survey results: Table 2 presents a summarised survey of the statistics by institution status, capacity and number of participants,

Table 2

Health care equipment maintenance specialist survey data - institutions and participants

\begin{tabular}{llcc}
\hline Institution status & Beds & $\begin{array}{l}\text { No. of } \\
\text { Institutions }\end{array}$ & Participants \\
\hline Small hospitals & $75-300$ & 2 & 4 \\
$\begin{array}{l}\text { Medium hospitals } \\
\text { Large Hospitals }\end{array}$ & $301-600$ & 2 & 10 \\
$\begin{array}{l}\text { Regional Maintenance } \\
\text { workshop }\end{array}$ & - & 4 & 16 \\
\hline Total & - & 2 & 8 \\
\hline
\end{tabular}

Results of this study were reviewed in the following categories: hospital size (number of beds); health care equipment maintenance; frequently used equipment with 
high maintenance demand; equipment in-house maintenance versus external maintenance service; equipment donations; spare parts inventory; equipment warranty; and recognition of clinical engineering department. The discussion of the results is comprehensively covered in the paragraphs below.

Health care equipment maintenance: The views of equipment maintenance specialists on equipment maintenance can be summarised as follows:

1. Health care equipment maintenance represented a problem to 34 (about 90\%) of the 38 respondents. (a) 20 indicated equipment maintenance was a major problem, (b) 10 indicated that it was a significant problem, (c) four indicated that it was an insignificant problem, (d) Health care equipment represented no problem to four of the respondents.

2. The significance of the maintenance problem increased with hospital size.

(a) Based on four hospitals of over 600 beds (large hospital): three of the participants claimed maintenance was a major problem and one a significant problem, (b) Based on two hospitals of 301-600 bed (medium hospitals): one participant claimed maintenance was a major problem and the other participant claimed it was a significant problem, (c) Based on two hospitals of between 75 and 300 beds (small hospitals): one participant claimed it was a significant problem while the other claimed maintenance was not a problem, (d) Based on two Regional Maintenance Workshops, all eight participants claimed equipment maintenance was becoming a significant problem because of vast changes in equipment design and inadequate training for maintenance staff on new equipment. Field visits and also face to face interviews with equipment maintenance experts confirmed that equipment maintenance problems were severe in larger hospitals.

Equipment maintenance budget: Inadequate maintenance budget was frequently mentioned by experts as a major problem in providing effective equipment maintenance in the public sector. Most claimed maintenance budgets were small and unrealistic. A survey on hospital budgets in 1999/ 2000 financial years, covering 54 hospitals $(75-300$ beds 25 hospitals; $300-600$ beds -16 hospital; $>600$ beds -13 hospitals), also showed that maintenance budget were inadequate.

The main characteristics prevalent in all hospital budgets in the hospitals covered in the survey were that staff salary budgets are consistently high (mean average of $70 \%$ ) and equipment maintenance budgets are consistently low (mean average of $3 \%$ ). The internationally recommended ratio of health care equipment budget to hospital budget is $10 \%$ cent(14).

Frequently used equipment with high maintenance demand: Equipment maintenance experts were asked to rank different equipment groups according to frequency of use and maintenance demands. All of the 38 respondents stated that diagnostic equipment is the most frequently used; monitoring equipment ranks second, followed by therapeutic equipment (third) and laboratory equipment (fourth). The frequency of use and hence maintenance demand of various types of equipment (Table 3 ).
Table 3

Health care equipment ranked by frequency of use and maintenance demand

\begin{tabular}{lcc}
\hline Equipment category & $\begin{array}{l}\text { No. of times } \\
\text { mentioned }\end{array}$ & \% of Total \\
\hline Diagnostic equipment & 42 & 40.38 \\
Electrocardiograph (ECG) & 20 & 19.23 \\
X-ray units & 16 & 15.38 \\
Others & 6 & 5.77 \\
Monitoring Equipment & 32 & 30.76 \\
Patient monitors & 18 & 17.30 \\
Cardiac monitors & 14 & 13.46 \\
Therapeutic equip. & 16 & 15.39 \\
Haemodialysis machines & 8 & 7.69 \\
Defibrillators & 4 & 3.85 \\
Others & 2 & 1.92 \\
Laboratory and other & & \\
equipments & 8 & 7.69 \\
Sterilizers & 6 & 5.77 \\
Others & 2 & 1.92 \\
Information and data & & 3.85 \\
systems & 4 & 2.89 \\
Computer printers paging units & 3 & 0.96 \\
Other & 1 & 100.00 \\
\hline Total & 104 & \\
\hline
\end{tabular}

The finding of diagnostic, monitoring and therapeutic equipment as groups of equipment with high maintenance demand was consisted with actual maintenance expenditures by three leading hospitals, namely, Kenyatta National Hospital, Nairobi; and Tugerberg and Groote Schuur Hospitals in Cape Town. They all showed high maintenance expenditures on diagnostic, therapeutic and laboratory equipment $(5,8,9,11)$. Figures 1 shows the trend of maintenance expenditures at Groote Schuur hospitals.

Figure 1

Health care equipment maintenance expenditure -1999/ 2000 financial year (GSH)

Courtesy: Clinical Engineering Department, Groote Schuur Hospital (GSH), Cape Town, 2000. 
Equipment donations: Out of 27 responses from hospitalbased specialists (30 in total) on the question of whether equipment donations were beneficial to the hospitals, 25 respondents claimed that most high-tech equipment donated to hospitals did not work beyond. providing positive media publicity to the "donor", and much of donated high-tech equipment often ends up in equipment store rooms (equipment funeral homes'). There are always some technical or budgetary problems associated with donated equipment. However, there were concurring views from 23 respondents that donation of simple devices like stethoscopes, scales, drip stands and basic surgical instruments were beneficial to all hospitals. Four respondents had no experience with donated equipment. Other studies have also provided negative appraisal to equipment donations $(4,10)$.

Equipment donation has remained a controversial issue in African countries. Despite charitable intentions of the donors, hospitals rarely benefit from donated equipment due to a variety of factors $(6,7)$. The World Bank Development Report of 1993, Investing in Health Care, concluded that even when donated equipment meets local requirements, very little of it ever becomes operational, for reasons - including missing or damaged parts, lack of disposable inputs and of user and service manuals, and problems with power supply. Another World Bank study concluded that the greatest obstacle to improving health technology in Africa was "technology philanthropy" - the uncoordinated donation of equipment to African countries by foreign agencies and charities. A study by Scottish Overseas Health Support recommended that African countries should have "donation protocols," whereby the kinds of equipment to be donated would follow a model paralleling, for example, the selection of drugs by using essential drugs lists (10).

Kachieng'a(4) concluded in his study that equipment donations should be treated as investments since recipient hospitals have to bear the costs of ownership (maintenance, consumables, training etc.). Therefore, it is perfectly legitimate to ask donors to accept various conditions in making their "gifts". Donations should not be used as a gentle way of dumping useless equipment in developing countries. A wrong donation is always more expensive than a rejected one.

In-house versus external maintenance service providers: Data supplied by the participants indicated that the eight hospitals were predominantly using a combination of internal and external maintenance services. The larger hospitals with well-trained and experienced maintenance personnel showed a tendency to rely more heavily on in-house maintenance service. The survey data showed that smaller hospitals depend heavily on external maintenance contractors, and the maintenance departments are poorly staffed. Maintenance problems and payments for contracts are mostly co-ordinated by hospitals' administration, and not maintenance departments.

The equipment specialists were in common agreement that there is a general need for an internal maintenance staff in all categories of hospitals, either to co-ordinate and supervise maintenance services provided by external maintenance providers or to carry out actual equipment maintenance work. The findings indicated that small hospitals (75-300 beds) rely more heavily on external maintenance contractors and warranties. Medium and larger hospitals depended less on warranties and used in-house service for general maintenance, only using external contractors for specialised repairs and services such as calibration and quality control protocols.
Spare parts inventory: Seventy percent of participants (experts) stated that they had an inventory of spare parts for some pieces of equipment. The importance of the inventory increased with hospital size (Table 4).

\section{Table 4}

Spare parts demand by hospital size

\begin{tabular}{lll}
\hline $\begin{array}{l}\text { Hospital size } \\
\text { (Beds) }\end{array}$ & $\begin{array}{l}\text { Participants } \\
\text { (experts) with } \\
\text { spare parts inventory }\end{array}$ & $\%$ of Total \\
\hline 75 up to 300 & 2 out of 4 & 50 \\
301 up to 600 & $\begin{array}{l}6 \text { out of } 10 \\
16 \text { out of } 16\end{array}$ \\
600 and over & 24 out of 30 & 70 \\
\hline Total &
\end{tabular}

Out of 23 responses from hospital-based specialists on the question of whether spare parts inventory in the hospital was deemed critical, 18 respondents stated that it was critical and five said it was not. The specialists from hospitals located far from major urban centres felt insecure without essential spare parts for critical diagnostic, therapeutic and laboratory equipment. The spare parts insecurity issue also surfaced in discussions during field visits to rural hospitals. The breakdown according to hospital size is presented in Table 5 .

\section{Table 5}

Response on stocking of spare parts

\begin{tabular}{llll}
\hline Hospital size & $\begin{array}{l}\text { Total no. of } \\
\text { responses }\end{array}$ & $\begin{array}{l}\text { Yes } \\
\text { responses }\end{array}$ & $\begin{array}{c}\text { No } \\
\text { responses }\end{array}$ \\
\hline $75-300$ & 2 & 1 & 1 \\
$301-600$ & 7 & 5 & 2 \\
$>600$ & 14 & 12 & 2 \\
\hline Total & 23 & 18 & 5 \\
\hline
\end{tabular}

Equipment warranty: Thirty-three respondents out of the 38 participants in the survey answered the question on whether the present warranties offered by suppliers were satisfactory. Eighteen of the respondents stated that they were; 11 said they were not, and four stated that it depended on the type of equipment. The reasons most commonly given for dissatisfaction with warranty policies are presented in Table 6 .

There were 35 responses to the question of whether warranties should continue to be offered. Twenty nine respondents stated that they should, while six said it would depend on the type of equipment. The specialists stated that better warranty conditions could enhance the ability of the hospitals to operate and maintain sophisticated equipment and devices more cost-effectively. They also noted that hospital administration, which normally negotiates warranty contracts, lacks technical expertise to negotiate broad-based warranty.

Warranties and services offered by manufacturers, suppliers and independent maintenance providers were viewed as inadequate by more than half of the participants. The inadequacies cited included limited time and parts coverage of warranties, poor workmanship by inadequately trained 
servicing personnel, and lengthy repair times, leading to high equipment downtime. It was evident during hospital visits and face to face interviews with experts that most hospitals do not have the technical expertise to negotiate appropriate warranty suitable for their situations. More often than not, they take warranty package offered by equipment suppliers without any appropriate amendment; later on the institutions blame the suppliers for unfair warranty agreement.

Table 6

Reasons for warranty dissatisfaction

\begin{tabular}{lll}
\hline Reason & $\begin{array}{l}\text { No. of times } \\
\text { mentioned }\end{array}$ & $\%$ of Total \\
\hline $\begin{array}{l}\text { Limited spare } \\
\text { parts coverage }\end{array}$ & 6 & 55 \\
$\begin{array}{l}\text { Poor workmanship } \\
\text { and service }\end{array}$ & 3 & 27 \\
Lengthy repair time & 2 & 18 \\
\hline Total & 11 & 100 \\
\hline
\end{tabular}

Recognition of clinical/biomedical engineering department: The responses on degree of recognition of clinical engineering departments by hospital administration fell into three categories: Full recognition, partial recognition and no recognition. Out of the total of 30 hospital based respondents, $13 \%$ indicated that their departments were sufficiently recognised, $17 \%$ achieved no recognition and $70 \%$ achieved partial recognition. Recognition was defined as attending departmental meetings, equipment pre-purchase consultations and consultation on other health technical support issues. The factors indicating non-recognition and partial recognition were the department being bypassed during the equipment acquisition process, while still being expected to maintain and repair equipment and devices. In such cases the departments are treated as "repair depots" and as such are not recognised or supported by hospital administration. A study of the correlation between the variable of 'level of prepurchase consultation' and recognition of the department yielded highly significant results. No recognition and partial recognition were concentrated in small and medium hospitals, while sufficient recognition of clinical engineering departments was most pronounced at large hospitals with sophisticated equipment.

\section{DISCUSSION}

In this section the trends emerging from the questionnaires are elaborated with further details gathered from the subsequent field interviews with equipment maintenance specialists.

Budgetary and infrastructural support: The specialists were of the opinion that support (infrastructure and budgetary) to health care equipment maintenance remains inadequate and many small and medium hospitals have not fully recognised equipment maintenance as a major contributing factor to efficient and cost-effective health care service delivery. Some large hospitals (meaning large urban and teaching hospitals) have recognised the importance of equipment maintenance and have accordingly employed trained clinical/medical engineers and technicians and established maintenance workshops and spare parts inventory management systems. However, most hospitals still lack adequate technical infrastructure and fiscal budgetary support to maintenance services. A frequent comment from the specialists was that maintenance contracts were generally negotiated by personnel of hospitals' administration or financial departments, who do not have the technical capacity to assess consequences and technical implications of the contracts.

Equipment selection and acquisition: The maintenance specialists felt that there is a need for equipment selection and procurement policy or guidelines. Inappropriately procured equipment is normally the beginning of maintenance problems, often rendering the equipment non-functional. According to the respondents, lack of equipment standardisation, poor management information support (inventory and asset management systems), poor logistics of spare parts and lack of ongoing training compound the problems of maintenance for technical personnel and users. This concurs with the study by the World Bank $(7,10)$, which concluded that standardisation could simplify management and maintenance and reduce inventory costs.

The evidence gathered from the survey and field visits showed that the participation of clinical engineering staff in equipment selection and procurement is generally limited to the writing of technical specifications. The limitation is greater in smaller hospitals than larger ones, but even in the larger hospitals the participation of clinical engineering staff is limited to facility level. On major equipment acquisitions clinical engineers are sometimes invited to take part in tender adjudication, but this is an exception rather than policy.

Management of equipment procurement: The specialists felt that there is a need for policy guidelines on equipment procurement. Well-managed equipment procurement can save both time and money, as a result of the shorter time required to train operating personnel and to install and commission equipment, lower frequency of breakdowns and accompanying inconvenience, shorter equipment downtime, smaller expenditure for parts and maintenance, and fewer preventive maintenance requirements. The equipment specialists in small hospitals considered their participation in equipment procurement unsatisfactory.

Equipment procurement committees and the tender board: The specialists felt that equipment procurement committees and the tender board should give careful attention to special requirements such as equipment calibration, standardisation, and quality control, since few hospitals have certified and qualified staff to provide these services. Compatibility of new equipment with existing equipment is important, both for safety and for ease of integration into existing diagnostic, therapeutic or patient monitoring systems. 
The standardisation of power sources, power connectors and other hardware leads to overall economy of operation and a higher degree of safety for the patient, despite a possible higher initial cost. Currently, the absence of government and industry standards has resulted in a variety of incompatible equipment connectors and devices that present potential safety hazards. Tender boards, as presently constituted, do not have the technical capacity to address all technical issues required for successful equipment selection and procurement $(4,9)$.

Equipment purchase price versus cost of ownership: The specialists felt that the tender boards focussed too much on purchase price, rather than the cost of ownership which encompasses all direct and indirect expenses associated with an item of health care equipment over its economic life. It is estimated that the purchase price represents only $20 \%$ of the cost of ownership(4). Careful consideration of all life-cycle costs is absolutely essential to hospital administrators in determining the best equipment for their purposes. What seems to be the most expensive equipment in the beginning may prove to be less costly in the long run $(2,7,11)$.

Equipment lease: Some of the equipment specialists (from South Africa) indicated that certain equipment suppliers have started equipment lease services, mainly in the private sector. It is a growing trend world-wide and an option worth considering, with advantages such as minimal initial capital outlay and quick availability $(4,6)$. It is also becoming a business practice in the private sector to outsource services like computing and information systems to private services providers.

The decisions whether to lease or buy depend on the situation in an institution. However they should be based on analysis of comparable break-even costs between lease cost, ownership cost and the percentage of time the equipment will be productively employed over its useful economic life-span. The cost of leasing is usually limited to the monthly fee (with in built component costs), whereas ownership costs include maintenance, calibration, depreciation and storage, as well as the cost of having capital tied up in the equipment.

The main obstacle to leasing equipment in the government hospitals is that the tender boards have no system for leasing equipment. Even upgrading equipment meets a strong bureaucratic opposition at the tender boards. Some hospitals opt for new equipment purchases, rather than go through lengthy approval procedures involved in equipment upgrades. The consequence is that fairly functional items of equipment requiring inexpensive upgrades are replaced by new ones just to avoid unnecessary bureaucratic red tape.

Equipment maintenance: The survey data indicate that maintenance programmes consisting of a combination of both internal and contracted maintenance services was the most common in public health facilities.
Specialists from large hospitals (16 experts) felt that there should be an "equipment alert information centre" where non-performing equipment can be reported or black-listed by clinical engineering departments. At present, equipment that has failed to perform in some public hospitals is sold to other unsuspecting public hospitals. The experts also felt that public hospitals should collaborate to share some essential maintenance services as is done in the private sector. They envisaged hospitals utilising a single or several specialised organisation(s) for equipment maintenance, calibration and training of maintenance staff. It was generally felt that state hospitals should use their 'market purchasing power' to negotiate for better maintenance contracts from external equipment maintenance service providers.

Equipment specialists felt that equipment manufacturers and suppliers control equipment maintenance through service contracts, and that they limit information about their products by providing poorly written service manuals. This lack of cooperation, from manufacturers, they felt, hinders cost-effective management of technology. Some suppliers also practice "sell and run" philosophy, where they do not provide after-sales back-up services. These practices impede effective transfer of health technology from industrialised countries to African countries.

Training of equipment users and operators: There was total agreement amongst all respondents that training of equipment users and operators reduces equipment downtime. The main problem is lack of training funds and personnel to carry out continuous or periodic training on equipment. All hospital-based respondents noted that nurses are the primary users of health care equipment, and many of them feel inadequately trained in equipment operation. In spite of continuous inter-departmental transfer of nurses, there is no ongoing regular training of nurses in their "new" departments. Many nurses (64 persons) interviewed during the field visits believed that their training does not prepare them adequately for new technologies. In some instances nurses had not even been trained on a new technology before using it. Kachieng'a(4) states that inadequate training of nurses, especially those working in the intensive care units merits special attention in Kenya and South Africa.

The equipment specialists group estimated that 20$30 \%$ of maintenance calls is user related. Many experts believe that the vast majority of equipment-related injuries are due to user error, rather than from equipment malfunction(4). According to the 38 equipment maintenance experts, training efforts that attempt to teach too much about electronics have not been successful, but programmes that stress proper use of specific equipment utilising a step-by-step approach have resulted in a dramatic reduction in service calls.

Equipment design trends and spare parts: The specialists noted that in the last decade equipment design has gone from a high proportion of mechanical 
components to a high proportion of electronic components. The life cycle of most electronic-based health care equipment has shrunk from 10 years a decade ago to five years presently, due to rapid innovations in health technology $(4,11,12)$. However, within the five years life span, the equipment still requires frequent upgrading (soft and hardware) to increase either efficiency or capacity. Equipment maintenance experts felt that hospital administrators and the tender boards should re-position themselves technically to match these new equipment life cycle trends in order to remain competitive.

Increased use of modular electronic elements in health care equipment will require that fewer types of replacement parts be stocked for repair and service, compared with the many individual components now required. Faults in equipment designed using modular electronic elements can usually be diagnosed more easily and equipment can be repaired and returned to proper operating conditions more quickly than equipment of traditional design. Even though a single component costs less than a modular element consisting of many components, and even though more sophisticated testing and calibration instruments are necessary, nevertheless the labour required to isolate and replace one faulty component, and the longer downtime involved, make equipment designed to use modular elements well worth consideration.

\section{CONCLUSION}

The data from this survey clearly suggest that, while health care equipment is one of the necessary components of a comprehensive health care system, pieces of equipment by themselves are scarcely sufficient without technical and budgetary support, including funding of training of both maintenance staff and equipment operators and users. Kenya and South Africa must develop human capital to manage their national health technological stock more efficiently, if the losses in technological investments are to be reduced. Technological investments should improve health outcomes, rather than diminish them.

Management of expensive and sophisticated equipment in larger hospitals merits special attention because of high technological investments in these institutions. Most of these institutions also serve as teaching hospitals for training medical personnel and clinical engineers. A maintenance and repair service has two basic actions in a hospital. One is to preserve technical operability and the second is to provide information essential for equipment management, especially for deciding about the selection and procurement of equipment. Both functions are still in their infancy in Kenya and South Africa.

Strategic planning of technological investments and efficient management of health care equipment are the key drivers in achieving affordable, accessible and cost-effective health services and better health outcomes. This requires not only the ability to understand and make the best use of recent scientific discoveries and technological innovations in medicine, but also optimal utilisation of existing technological resources in health care services. Strategic planning and appropriate policies for the management of technological investments is currently the most challenging task to public health policymakers and planners in Kenya and South Africa, if the goal of delivering better health care services to all the population while containing the rapidly escalating costs is to be achieved.

\section{RECOMMENDATIONS}

On the basis of the results of the survey presented in this study, the following recommendations are made: (i) There is need to strengthen and streamline management of the technical infrastructure for health care equipment selection, procurement and maintenance management. Equipment maintenance budgets should be linked to actual equipment capital investment and rate of utilisation. Hospitals should not purchase equipment, which their maintenance budgets cannot support.

(ii) Hospitals need equipment assets management systems for monitoring equipment life-cycle costs, maintenance costs and management of equipment replacement. A standard equipment list (SEL) should be compiled for hospitals of different sizes. The SEL should guide investments on essential equipment.

(iii) The operations of the tender board need to be redefined. Under the present tender conditions, there are no incentives or provisions for public hospitals to seek discounts and warranty credits for major equipment purchases. Secondly, although the tender board is the budget holder, it is the hospitals that carry the burden of equipment ownership and pay the economic penalties for inappropriate acquisitions, not the tender boards; therefore they should have more say in equipment acquisitions through the tender boards. New tender Board guidelines and protocols to facilitate equipment upgrades and lease should be compiled.

(iv) To achieve optimum utilisation of expensive equipment, specialised departments in public hospitals, such as radiation therapy, radiology, nuclear medicine and renal units, should be permitted to provide specialised technological services to private patients 'after hours' to generate funds for equipment maintenance and replacement and for staff training. A fund distribution formula of 5030-10-10 can be used, where $50 \%$ of the funds generated remains in the mother department, $30 \%$ goes to the feeder departments, $10 \%$ to hospital administration and the final $10 \%$ to the government. The essence of this recommendation is to assist public hospitals to generate their own funds for equipment maintenance rather than depending on inadequate equipment maintenance budgets provided by the government. 


\section{REFERENCES}

1. Emergency Care Research Institute (ECRI) (1989). Technology Management: Preparing Your Hospital.for the 1990s. Health Technology, 1989; 3:111-115.

2. Bronzino, J.D. (ed.). The Biomedical Engineering Hand Book. Washington: IEEE Press. 1995.

3. David, Y. and Judd, T.M. Medical Technology Management. Biophysical Measurement Series. Washington: Space Labs Inc. 1993.

4. Kachieng'a, M.O. Health technology assessment in subSaharan Africa: A cross national study of Kenya and South Africa. Doctorate Thesis in Biomedical Engineering, University of Cape Town. 1999.

5. Kachieng'a, M.O. An African Safari in health technology - From Cape Town to Nairobi in 10 Days. South Afr. Med. J. 2002; 92:344-345.

6. Kachieng'a, M.O. and Boonzaier, D.A. Health care technology assessment: South African health care system in transition. South Afr. Med. J. 1999; 89:149-155.

7. World Bank. Investing in Health. World Development Report 1993. The World Bank Publication. New York: Oxford University Press; 1993.

8. Kachieng'a M.O. Health Care Technology in Public Health Care Institutions in Kenya. East Afr. J. Med. 1998; 75:632636.

9. Ncayiyana, D. The equity gap debate (Editorial). South Afr. Med. J. 1997; 5:87.

10. World Bank. Better Health in Africa: Experiences and Lesions Learned. The World Bank Publication. New York: Oxford University Press; 1995.

11. Kachieng'a, M.O., Boonzaier D. A., Fataar A.B., Boniaszczuk $\mathrm{J}$ and Boltman, G. Assessing the use of nuclear medicine technology in Sub-Saharan Africa: The essential equipment list. J. Nuclear Med. Tech. 1999; 27:1-5.

12. Pacela, A.F. Survey of salaries and responsibilities for hospital biomedical/clinical engineering and technology personnel. J. Clin. Eng. 1992; 17:215-231. 